widrigkeitenrecht. Das Rechtsmittel ist dann nicht die Strafe, sondern eine Geldbuße. Um zu prüfen, ob eine Ordnungswidrigkeit vorliegt, muss man erneut an das Ende der Gesetze schauen. Im Fall des MPG schließen sich die Regelungen $z u$ den Geldbußen direkt an die zur Strafbarkeit an, sie stehen in $\$ 42$ MPG. Und tatsächlich heißt es dort in Absatz 2: „Ordnungswidrig handelt, wer vorsätzlich oder fahrlässig 1. entgegen $\$ 4$ Abs. 1 Nr. 2 ein Medizinprodukt in den Verkehr bringt, errichtet, in Betrieb nimmt, betreibt oder anwendet, $[. . .]^{\text {“. }}$

Das bedeutet: Bei abgelaufenem Datum von Medizinprodukten muss der Betreiber oder Anwender nicht mit einer Strafe rechnen, aber mit einer Geldbuße.

\section{Voraussetzungen müssen erfüllt sein}

Doch zurück zur Strafbarkeit. Ist eine Vorschrift gefunden, die eine bestimmte Pflichtwidrigkeit tatsächlich unter Strafe stellt, dann müssen nunmehr akribisch all deren Voraussetzungen geprüft werden. Erst wenn sie alle bejaht werden können, dann ist die Pflegekraft auch tatsächlich strafbar. Bei einer vorsätzlichen Tat bedeutet das:

_Liegen alle objektiven Tatbestandsmerkmale vor? Wurde z.B. im Falle einer Freiheitsberaubung nach $\$ 239$ StGB auch tatsächlich die Freiheit entzogen? Was z.B. dann ausgeschlossen wäre, wenn bei einem Bettlägerigen, der sich nicht mehr willentlich bewegen kann, zu dessen Schutz Bettseitenteile angebracht werden (um ihn vor dem unwillkürlichen Herausfallen aus dem Bett zu bewahren).

- Kannte die Pflegekraft alle Umstände des objektiven Tatbestands?

_ Gibt es Rechtfertigungsgründe? So wäre die Strafbarkeit beispielsweise ausgeschlossen, wenn der Betreuer in das Anbringen von Bettseitenteile eingewilligt hat (Einwilligung als Rechtfertigungsgrund). Oder wenn der Patient akut selbstgefährdet war (rechtfertigender Notstand).

_Und schließlich: Ist die Tat der Pflegekraft auch wirklich persönlich vorzuwerfen? Gab es womöglich Entschuldigungsgründe?

Wird einer Pflegekraft nicht vorgeworfen, dass sie aktiv tätig geworden ist, son- dern vielmehr, dass sie gerade nicht gehandelt hat, obwohl sie es hätte tun müssen, dann muss zusätzlich die Garantenstellung der Pflegekraft geprüft werden. Hatte die Pflegekraft in der konkreten Situation tatsächlich die Pflicht, etwas zu unternehmen?

Bei Fahrlässigkeitsdelikten kommt es vor allem darauf an, ob der Pflegekraft eine Sorgfaltspflichtverletzung zur Last gelegt werden kann. Und ob der „Erfolg“ objektiv vorhersehbar war. War es also z.B. für die Pflegekraft wirklich vorhersehbar, dass der Betroffene stürzt, wenn die Pflegekraft ihn für einen kurzen Moment alleine stehen lässt?

Wenn eine versuchte Tat bestraft werden soll, dann stellt sich immer wieder

\title{
RICHTIGES VERHALTEN IM SCHADENSFALL
}

Zunächst sollten Sie nach einem (nicht nur strafrechtlich relevanten) Vorfall einen Bericht für den eigenen Gebrauch schreiben. Erledigen Sie das möglichst schnell, weil Ihre Erinnerungen dann noch frisch und weitgehend vollständig sind. Dieses Protokoll hilft Ihrem Gedächtnis später auf die Sprünge. Außerdem können Sie es als Grundlage für einen offiziellen Schadensbericht verwenden. Denken Sie bei letzterem daran, den relevanten Vorgang möglichst neutral zu schildern. Auf keinen Fall sollten Sie ein Schuldeingeständnis abgeben.

Sichern Sie möglichst viele Beweise: Suchen Sie Zeugen, machen Sie Fotos von Örtlichkeiten und Kopien von wichtigen Dokumenten. Vergessen Sie dabei nicht, gegebenenfalls die dazu notwendige Erlaubnis einzuholen. Den direkten Kontakt zum Geschädigten sollten Sie möglichst einschränken oder - wenn möglich - sogar völlig vermeiden. So können Sie einer weiteren Eskalation aus dem Weg gehen und verhindern, dass Sie Informationen weitergeben, die nachher womöglich auf sie zurückfallen.

Wenn Sie von der Polizei befragt werden, dann sollten Sie keine Angaben zur Sache machen. Sie sind lediglich verpflichtet, über ihre Person (Name, Geburtsort und -datum etc.) Auskunft zu geben. Lassen Sie sich weder "einwickeln" noch „bedrohen“. Vermeiden Sie auch Spontanäußerungen oder "nette Unterhaltungen”.

Werden Sie zur Polizei geladen, müssen Sie dort in der Regel nicht erscheinen. Wohl aber vor einem Staatsanwalt oder Ermittlungsrichter. Allerdings haben Sie auch dort das Recht, Angaben zur Sache zu verweigern:

— Als Zeuge haben Sie zunächst das Recht, eine Aussage generell zu verweigern. Zum Beispiel dann, wenn ein naher Angehöriger beschuldigt wird ( $\$ 52$ Strafprozessordnung, StPO). Aber auch als sogenannter „Berufshelfer" von Ärzten ( $\$ 53 a$ StPO). In diesem Fall entscheidet der Arzt, ob Sie zu einem medizinischen Sachverhalt eine Aussage machen dürfen. Selbstverständlich kann Sie auch der Patient von der Schweigepflicht entbinden.

_ Das Recht, auf bestimmte Fragen die Auskunft zu verweigern, haben Sie als Zeuge dann, wenn Sie bei der Beantwortung Gefahr laufen würden, damit sich selbst oder Angehörige der Verfolgung wegen einer Straftat oder einer Ordnungswidrigkeit auszusetzen.

_ Werden Sie nicht bloß als Zeuge, sondern gar als Beschuldigter befragt, dann dürfen Sie die Aussage in jedem Fall komplett verweigern.

Bei einer Durchsuchung und/oder Beschlagnahme: Lassen Sie sich den Durchsuchungsbefehl zeigen und prüfen Sie, ob die Durchsuchung von diesem auch gedeckt ist. Sie dürfen bei der Durchsuchung anwesend sein und auch einen Anwalt oder einen Zeugen hinzuziehen. Wenn möglich, dann helfen Sie den Beamten. Dadurch verkürzen Sie die Durchsuchung und verhindern größere Unordnung. Bei der Beschlagnahme sollten Sie sich eine möglichst detaillierte und exakte Quittung ausstellen lassen. Verlangen Sie bei beschlagnahmten Dokumenten, davon Kopien machen zu dürfen. Will die Polizei einen Computer mitnehmen, bitten Sie darum, eine Spiegelung der Festplatte vorzunehmen. Spätestens, wenn Sie zur Staatsanwaltschaft oder vor den Ermittlungsrichter geladen werden oder wenn bei Ihnen durchsucht wird, sollten Sie unbedingt einen Rechtsanwalt einschalten.

Thorsten Siefarth, Rechtsanwalt 